\title{
El docente en las Aulas Hospitalarias Españolas. Un estudio comparado del perfil profesional específico en el marco autonómico español
}

\author{
Asensio-Ramón, Patricia \\ Universitat de València, Valencia, España \\ patricia.asensio@uv.es
}

\section{Resumen}

La Pedagogía Hospitalaria, sus aulas y, todo el equipo que forma parte de ellas, está avanzando a pasos agigantados por ir más allá del paciente y de las plantas de pediatría, involucrando así a todo un equipo interdisciplinar.

Con la Organización Mundial de la Salud (OMS) a través de un grupo de estudio trató el tema de la Educación Multiprofesional dentro del ámbito sanitario con profesionales que tratan tareas que benefician la salud, como la prevención, curación, rehabilitación. Gracias a ello hay numerosos estudios que admiten que, para que el ingreso sea lo menos traumatizante posible es imprescindible que el niño-paciente lleve una vida lo más normalizada posible.

El objetivo de nuestra propuesta ha sido mostrar las peculiaridades de esta disciplina y, realizar un estudio comparado de uno de los indicadores que forman parte de este ámbito educativo: la titulación específica exigida a los profesionales que imparten docencia en dichas Aulas Hospitalarias.

Mediante este estudio quedará reflejado si las competencias educativas propias de las Comunidades Autónomas españolas muestran variabilidad dentro de las Aulas Hospitalarias del sistema educativo español. Para realizar este estudio la metodología ha sido primeramente, un estudio cualitativo de la titulación específica exigida en cada Comunidad Autónoma objeto de estudio. Seguidamente, se ha realizado una yuxtaposición de resultados para, a continuación, elaborar un estudio comparado de dicho indicador en las autonomías seleccionadas por su trayectoria histórica y desarrollo normativo. Finalmente, se han realizado unas consideraciones mostrando las convergencias y divergencias existentes entre ellas y finalizando con una pequeña reflexión.

\section{Abstract}

Hospital Pedagogy, its classrooms, and all the team that is part of them, is advancing by leaps and bounds to go beyond the patient and the pediatrics plants, thus involving an entire interdisciplinary team.

With the World Health Organization (WHO) through a study group he addressed the issue of Multiprofesional Education within the health field with professionals who treat tasks that benefit health, such as prevention, cure, rehabilitation. Thanks to this there are numerous studies that admit that, in order for the hospital to be the least traumatic, it is essential that the patient-child lead a normal life as possible.

The objective of our proposal has been to show the peculiarities of this discipline and to carry out a comparative study of one of the indicators that are part of this educational field: the specific qualification required of the professionals who teach in said Hospital Classrooms. Through this study will be reflected if the educational competencies of the Spanish Autonomous Communities show variability within the Hospitals Classrooms of the Spanish educational system.

To carry out this study, the methodology was first a qualitative study of the specific qualification required in each Autonomous Community under study. Next, a juxtaposition of results has been made to then elaborate a comparative study of said indicator in the Autonomies selected for their historical trajectory and normative development. Finally, some considerations have been made showing the convergences and divergences between them and a small reflection to make a proposal for improvement within this field of educational inclusion.

Palabras clave: Aulas Hospitalarias, Perfil, Comunidades Autónomas.

Keywords: Hospital Classrooms, Profile, Autonomous Communities. 


\section{INTRODUCCIÓN}

Las Aulas Hospitalarias surgieron ante la necesidad de resolver los problemas de escolaridad de aquellos niños que debido a una enfermedad no podían asistir de forma regular a la escuela. A lo largo de los años y, gracias a estos espacios físicos ubicados en los hospitales, se ha conseguido continuar con el proceso normal de enseñanza y aprendizaje del paciente pediátrico.

Estas unidades escolares hospitalarias están definidas por diversos indicadores que componen la legislación que las regula. El estudio comparado de uno de ellos, en este caso el perfil profesional específico de los docentes que desarrollan el proceso de enseñanza-aprendizaje en dichas aulas, nos va a permitir comprobar si las competencias educativas de las que disponen las diferentes Comunidades Autónomas (CC.AA.) españolas presentan versatilidad dentro del sistema educativo español, pero especialmente dentro de los Programas de Atención Educativa Hospitalaria.

Para realizar dicho estudio se ha seleccionado diversas CC.AA. españolas distinguidas específicamente, por su ubicación socio-demográfica, su trayectoria dentro de la Pedagogía Hospitalaria y su historia ideológica.

\section{Objetivos}

El objetivo de nuestra propuesta ha sido mostrar las peculiaridades de esta disciplina y realizar un estudio comparado de uno de los indicadores que forman parte de este ámbito educativo: la titulación específica exigida a los profesionales que imparten docencia en dichas Aulas Hospitalarias.

Mediante este estudio quedará reflejado si las competencias educativas propias de las Comunidades Autónomas españolas muestran variabilidad dentro de las Aulas Hospitalarias del sistema educativo español.

\section{Metodología}

Para realizar dicho estudio, la metodología empleada ha sido primeramente, un estudio cualitativo de la titulación específica exigida en cada Comunidad Autónoma objeto de estudio.

A continuación, se ha realizado una yuxtaposición de resultados para, seguidamente, elaborar un estudio comparado de dicho indicador entre las autonomías seleccionadas. Finalmente, se han realizado unas consideraciones mostrando las convergencias y divergencias existentes entre ellas y finalizar con una pequeña reflexión.

\section{MARCO TEÓRICO}

\subsection{Las Aulas Hospitalarias}

Todo niño hospitalizado es un sujeto vulnerable, frágil que, requiere cuidados y atención de los familiares y agentes sociales que le rodean. Hemos de paliar sus necesidades, sus sufrimientos así como, descubrir sus posibilidades existenciales dentro de la personalidad que puede desarrollar. Es evidente, que en el contexto hospitalario siempre se deben asumir las necesidades del otro y el desarrollo máximo de sus posibilidades (Torralba, 2002).

Han sido múltiples las aportaciones que han definido las Aulas Hospitalarias como aulas que cubren las necesidades y, que dan respuesta al Derecho a la Educación de todo infante que no asiste a su centro escolar por una enfermedad, ingreso hospitalario o convalecencia. 
Para señalar los objetivos propios de dichas unidades escolares hemos seguido a Florez (2015), quien señala:

- Coordinar tareas de diferentes profesionales que intervienen en la educación del alumnado enfermo: centro docente, atención educativa domiciliaria, etcétera.

- Emplear el tiempo libre en el hospital.

- Buena integración del alumnado enfermo al centro educativo.

\subsection{El docente hospitalario: el perfil profesional específico según la Comunidad Autónoma española}

El objetivo principal de esta comunicación ha sido definir el perfil profesional específico de los docentes que trabajan en las Aulas Hospitalarias de las comunidades seleccionadas. Por otra parte, hemos comprobado si las competencias que dispone cada autonomía se reflejan en la variabilidad de dicho indicador. Para desarrollar dicho objetivo se ha hecho uso de la normativa y/o de las aportaciones docentes, para a continuación realizar una yuxtaposición y, posteriormente una comparación, de las autonomías objeto de estudio.

\subsubsection{El perfil profesional específico en el País Vasco}

El País Vasco presenta la peculiaridad de disponer de un Decreto 266/2006 que ha dado la posibilidad de poder crear Centros Territoriales para la Atención Educativa Hospitalaria, Domiciliaria y Terapéutico-Educativa (CAHDT-E). Sí que es cierto, que en dicho Decreto se describe cómo se organizan y funcionan dichos centros, sin embargo, no hace mención del perfil profesional específico requerido a los docentes que ejercen en dicho sector. Por consiguiente, se ha tenido que recurrir a las aportaciones ofrecidas por los propios docentes hospitalarios, así como el personal de dirección de los CAHDT-E.

La manera de acceder al puesto supone estar en posesión de plaza en propiedad y por comisión de servicios. El perfil profesional demandado es personal con titulación en Magisterio con la especialidad en Pedagogía Terapéutica (PT). Por otra parte, tanto la formación inicial como permanente en educación hospitalaria es inexistente.

\subsubsection{El perfil profesional específico en la Comunidad Valenciana}

La Comunidad Valenciana se rige por las Instrucciones del 18 de agosto de 2017 por la que se regulan las Unidades Pedagógicas Hospitalarias (UPH) para el curso 2017-2018. Por otra parte, también se basan en la Orden de 4 de julio de 2001 de la Consejería de Cultura, Educación y Deporte por la que se rige la atención al alumnado con necesidades de compensación educativa. En dicha Orden se especifica que dichos puestos serán cubiertos por funcionarios con la titulación de maestros y profesores de la Enseñanza Secundaria Obligatoria (ESO).

De igual forma que la Autonomía anterior, en la Comunidad Valenciana no se especifica el perfil profesional específico que debe poseer todo profesorado que ocupe dichos puestos. La información de dicho perfil se ha extraído de entrevistas realizadas a los propios docentes de dichas UPH. Se accede al puesto teniendo plaza en propiedad y mediante comisión de servicios. Por otra parte, especifican que el perfil académico demandado es titulado en Magisterio con la especialidad en Pedagogía Terapéutica (PT). Respecto a la formación inicial y permanente indican que es contradictoria a la señalada por la normativa, ya que la formación inicial por parte de los Servicios Psicopedagógicos Escolares (Spes) respecto a la Pedagogía Terapéutica, es inexistente. Finalmente, la formación permanente en PT ofertada por los Servicios de Formación del Profesorado de la Comunidad Valenciana (CEFIRE) es nula. Sí que podemos confirmar la existencia de seminarios en el sector elaborados por los propios docentes hospitalarios. 


\subsubsection{El perfil profesional específico en la Comunidad de Madrid}

En la Comunidad de Madrid pese a la existencia de unas instrucciones elaboradas específicamente para regular estas Unidades Escolares de Apoyo, Instrucciones de la Dirección General de Educación Infantil y Primaria para el funcionamiento de las Unidades Escolares de Apoyo en instituciones hospitalarias de la Comunidad de Madrid de 10 de diciembre de 2010, no queda reflejado el perfil profesional concreto demandado para impartir docencia en dichas unidades. Sin embargo, y como se ha realizado en autonomías mencionadas anteriormente, se ha recurrido a las aportaciones ofrecidas por docentes que ejercen en dichos puestos. Siguiendo a dichos profesionales del sector indican que se accede a ellos, siendo personal funcionario, teniendo plaza en propiedad y por comisión de servicios a través de una selección de currículum. Por consiguiente, se requiere la titulación de Magisterio con distintas especialidades según necesidad, es decir, podrán acceder profesionales especialistas en Primaria, Infantil, Educación Especial, Audición y Lenguaje, etcétera. Respecto a la formación inicial, hasta hace unos años en el mes de septiembre se realizaban unas jornadas sobre Aulas Hospitalarias para todo profesorado novel. Actualmente, se realizan de manera voluntaria, tanto como formación inicial como formación permanente para el profesorado veterano. Sí que es cierto, que en marzo de 2018 se ha realizado el I Congreso Internacional y IV Jornadas de Pedagogía Hospitalaria donde se han intercambiado experiencias tanto a nivel nacional como internacional.

\subsubsection{El perfil profesional específico de Extremadura}

La Comunidad extremeña se caracteriza por disponer de unas Instrucciones 2/2015 que concretan aspectos de atención a la diversidad según lo establecido en el Decreto 228/2014 donde se regula la respuesta educativa a la diversidad. Sin embargo, dicha autonomía carece de Instrucciones específicas dedicadas al servicio educativo hospitalario. Por tanto, para llegar a conocer el perfil profesional específico demandado al personal docente para cubrir estos puestos, se ha recurrido a las aportaciones ofrecidas por una Memoria de Actuación elaborada por el propio profesorado de las aulas y un plan de Actuación durante el curso 2012-2013.

Dicha documentación señala que para acceder a dichos puestos se ha de tener plaza en propiedad y se accede por comisión de servicios, ya que como en el resto de autonomías dichos puestos no salen a plaza por oposición. Por otra parte, independientemente del resto de titulaciones académicas, se exige la titulación de Magisterio con la especialidad en Pedagogía Terapéutica y/o Audición y Lenguaje.

Respecto a la formación inicial y permanente hay constancia de un seminario sobre Aulas Hospitalarias creado por el Centro de Profesores y Recursos de Badajoz.

\subsubsection{El perfil profesional específico en Andalucía}

Finalmente, Andalucía se caracteriza por disponer de unas Instrucciones destinadas a la Organización y el Funcionamiento de las Aulas Hospitalarias elaboradas en 2007. En dicha normativa señala que, dichos puestos docentes se cubren por convocatoria pública y el profesorado seleccionado lo ocupará por comisión de servicios. Sin embargo, dicha legislación no especifica el perfil profesional específico que debe poseer dicho docente hospitalario. Para disponer de esta información se ha recurrido a las aportaciones docentes que señalan que, el perfil específico demandado ha sido maestro de Infantil y Primaria, aunque sí que es cierto que, en alguna convocatoria se ha recurrido a la especialidad en PT.

Respecto a la formación inicial y permanente relacionada con el sector educativo hospitalario indican que es inexistente. Sí que se han organizado jornadas de encuentro entre profesorado de diversas Aulas Hospitalarias. 


\subsection{Análisis comparado del perfil profesional específico demandado en las Comunidades objeto de estudio.}

Como se ha podido observar, nos situamos dentro del ámbito de la Pedagogía Hospitalaria, aunque el profesional que ejerce funciones en uno de sus sectores, las Aulas Hospitalarias, no es el pedagogo sino el docente hospitalario.

Por otra parte, se ha podido comprobar que, en ninguna normativa de las autonomías seleccionadas que regulan dichas unidades, se refleja cuál es la especialidad demandada para ejercer en dichas unidades escolares.

A continuación, se ha elaborado la Tabla 1 donde se han yuxtapuesto los datos relevantes al perfil profesional específico demandado en cada autonomía objeto de estudio. Con los datos expuestos se han realizado unas consideraciones y conclusiones comparativas útiles para una reflexión final respecto al tema en cuestión.

Tabla 1. Perfil profesional específico demandado al profesorado hospitalario.

\begin{tabular}{|c|l|}
\hline Comunidades Autónomas & Perfil profesional específico demandado según CC.AA. \\
\hline País Vasco & Titulación de Magisterio con la especialidad en Pedagogía Terapéutica (PT). \\
\hline Comunidad Valenciana & Titulación de Magisterio con la especialidad en Pedagogía Terapéutica (PT). \\
\hline Comunidad de Madrid & $\begin{array}{l}\text { Titulación de Magisterio con distintas especialidades según necesidad: Primaria, Infantil, } \\
\text { Educación Especial, Audición y Lenguaje (AL). }\end{array}$ \\
\hline Extremadura & Titulación de Magisterio de Infantil y Primaria y, en alguna convocatoria, en PT. \\
\hline Andalucía & Titulación de Magisterio en Infantil y Primaria y, en alguna convocatoria en PT. \\
\hline
\end{tabular}

Fuente: elaboración propia.

\section{CONSIDERACIONES Y CONCLUSIONES COMPARATIVAS}

Tras haber descrito los datos relevantes sobre el tema que abordamos en esta comunicación, el perfil profesional específico demandado en las Aulas Hospitalarias de las autonomías objeto de estudio y, elaborar la Tabla 1 con los datos yuxtapuestos, se han obtenido una serie de consideraciones que han determinado el apartado de la conclusión comparativa. En este último punto se ha realizado una descripción de las semejanzas y diferencias observadas finalizando con una serie de reflexiones concluyentes.

Queda reflejado que en la normativa de las cinco autonomías objeto de estudio no queda señalado el perfil profesional específico demandado para ejercer como docentes en las Aulas Hospitalarias. Dicha información se ha obtenido en todas las autonomías seleccionadas gracias a las entrevistas realizadas al profesorado en cuestión.

En la Comunidad Valenciana, País Vasco y Extremadura se demanda el título de Magisterio en Pedagogía Terapéutica y, AL en el caso extremeño. Sin embargo, tanto en las Comunidades de Madrid y Andalucía conviven especialidades como infantil, Primaria, PT o AL dependiendo de la necesidad.

Es evidente y, queda mostrado en dicho indicador, que el perfil profesional específico para acceder a dichos puestos no está homogeneizado en las Comunidades objeto de estudio. Por tanto, manifestamos que es una de las carencias a destacar en la normativa reguladora de dicha disciplina en las cinco autonomías objeto de estudio.

Finalmente, se han podido establecer ciertas semejanzas entre dichas Comunidades Autónomas a través de este indicador. Gracias a estas comparaciones se han podido considerar las carencias establecidas en la normativa mencionada respecto a la variable, perfil profesional específico demandado para ejercer en las Aulas Hospitalarias españolas. Gracias a las entrevistas realizadas y, contribucio- 
nes ofrecidas por los docentes que llevan a cabo dicho servicio, se ha podido evidenciar que no existe una homogeneización respecto al criterio especialidad exigida según las Autonomías seleccionadas.

Por consiguiente y, de manera concluyente, en las cinco Comunidades objeto de estudio queda reflejado que, para acceder a dichos puestos se accede de manera similar, teniendo plaza en propiedad y por comisión de servicios. Sin embargo, las diferencias vienen marcadas por la especialidad exigida para ocupar dicho puesto marcando así, las competencias educativas de las que tiene cada autonomía.

\section{REFERENCIAS BIBLIOGRÁFICAS}

Fuentes bibliográficas

Arrieta, M. (2014). Pedagogía Hospitalaria, campo y objeto de estudio. En J. González (Coord.), Memorias I Congreso Internacional de Pedagogía Hospitalaria en Colombia (pp.88-90). Bogotá, Colombia.

Cardone, P., \& Monsalve, C. (2010). Pedagogía Hospitalaria: Una propuesta educativa de FEDUPEL. Caracas, Venezuela: Fondo Editorial de la Universidad Pedagógica Experimental Libertador.

Casas, C.,Corchero, C.,\& García, M.M.(2013).Memoria de actuación de las Aulas Hospitalarias de Badajoz, $2012-2013$. Unidad de Programas Educativos de badajoz. Gobierno de Extremadura. Recuperado de http://gset.net/index.php/ component/flexicontent/44-aula-hospitalaria/481-aulahospitalaria el 14 de febrero del 2015.

Casas, C., Corchero, C., \& García, M.M. (2014). Plan de Actuación Anual de las Aulas Hospitalarias de Badajoz, $2012-2013$. Unidad de Programas Educativos de Badajoz. Gobierno de Extremadura. Recuperado de http://gset.net/ index.php/component/flexicontent/44aulahospitalaria/481-aulahospitalaria el 14 de febrero del 2015.

Grau, C. \& Ortiz, C. (2001). La Pedagogía Hospitalaria en el marco de una educación inclusiva. Málaga: Aljibe.

Guillén, M. \& Mejía, A. (2002). Actuaciones educativas en aulas hospitalarias. Atención escolar al niño enfermo. Madrid: Narcea.

Latorre, M.J. \& Blanco, F.J. (2010). Función profesional del pedagogo en centros hospitalarios como ámbitos educativos excepcionales. Educación XXI, 13(2), pp.95-116.

Lieutenant, C. (2006). La evolución de las aulas hospitalarias. Un camino por recorrer. En S. Riquelme (Presidencia). Aulas Hospitalarias. Reflexiones sobre la VIII Jornada de Pedagogía Hospitalaria. Fundación Carolina Labra Riquelme, Chile.

Lizasoáin, O. (2000). Educando al niño enfermo. Perspectivas de la Pedagogía Hospitalaria. Pamplona: Ediciones Eunate.

Fuentes Legislativas

Instrucciones sobre la Organización y Funcionamiento de las Aulas Hospitalarias en la Comunidad Autónoma de Andalucía, 29 de junio de 2007.

Instrucciones de la Dirección General de Educación Infantil y Primaria para el funcionamiento de las Unidades Escolares de Apoyo en Instituciones Hospitalarias de la Comunidad de Madrid, 9 de diciembre de 2010.

Instrucción 2/2015, donde se concretan aspectos de Atención a la Diversidad según lo establecido en el Decreto 228/2014, de 14 de octubre, por el que se regula la respuesta educativa a la diversidad en la Comunidad Extremeña, 29 de enero de 2015.

Instrucciones del 18 de agosto de 2017 de la Dirección General de Política Educativa por la que se regula el funcionamiento de las Unidades Pedagógicas Hospitalarias ubicadas en hospitales públicos de la Comunidad Valenciana para el curso 2017/2018.

Orden de 4 de julio de la Consejería de Cultura, Educación y Deporte por la que se regula la atención al alumnado con necesidades de compensación educativa. DOGV, No. 4.044, 19.282 (2001). 